\title{
Les Trinci à Nocera. Mise en scène et construction de la violence dans une seigneurie italienne du premier Quattrocento
}

Jean-Baptiste Delzant

\section{OpenEdition}

Journals

Édition électronique

URL : http://journals.openedition.org/questes/1643

DOI : $10.4000 /$ questes.1643

ISSN : 2109-9472

Éditeur

Les Amis de Questes

Édition imprimée

Date de publication : 15 mars 2008

Pagination : 62-75

ISSN : 2102-7188

\section{Référence électronique}

Jean-Baptiste Delzant, «Les Trinci à Nocera. Mise en scène et construction de la violence dans une seigneurie italienne du premier Quattrocento », Questes [En ligne], 14 | 2008, mis en ligne le 01 janvier 2014, consulté le 30 avril 2019. URL : http://journals.openedition.org/questes/1643 ; DOI : 10.4000/ questes. 1643 


\section{Les Trinci à Nocera. \\ Mise en scène et construction de la violence dans une seigneurie italienne du premier Quattrocento ${ }^{1}$}

\section{Jean-Baptiste DELZANT}

La légende noire des tyrans italiens de la Renaissance a très tôt été construite. Les chroniqueurs $\mathrm{du} \mathrm{XV}^{\mathrm{e}}$ siècle ont recueilli, développé et transmis des anecdotes sanglantes sur les seigneurs dépravés du temps. Elisabeth Crouzet-Pavan l'a remarqué,

il y aurait pour chacune des familles italiennes, Visconti de Milan, da Carrara de Padoue, Trinci de Foligno ou Baglioni de Pérouse, une recherche à conduire qui montrerait comment, des chroniqueurs contemporains, favorables ou hostiles, aux monographies urbaines de l'époque moderne, une vulgate faite de légendes et de récits plus ou moins inventés, a été construite ${ }^{2}$.

Le cas des Trinci de Foligno permet de proposer quelques éléments de réponse à une telle invitation. Plusieurs textes écrits autour du second tiers du Quattrocento font en effet référence au même événement, d'une violence inouïe, advenu en janvier 1421. À travers le récit qu'ils en donnent, ils élaborent l'image fragmentée d'un seigneur pour lequel la violence est un instrument de pouvoir. L'interprétation proposée par chacun des auteurs s'insère dans une stratégie argumentative dont il s'agit de déceler les enjeux.

Le seigneur de Nocera, une petite ville d'Ombrie placée sous la domination des maîtres de Foligno, attire ces derniers dans un traquenard. Deux

\footnotetext{
${ }^{1}$ Mesdemoiselles Camille Delzant et Mélanie Adda ont relu avec attention cet article et l'ont enrichi par leurs suggestions. Qu'elles en soient chaleureusement remerciées.

${ }^{2}$ Élisabeth Crouzet-PAVAn, Renaissances italiennes, 1380-1500, Paris, Albin Michel, 2007, p. $145-146$.
} 
des trois frères Trinci, Niccolò et Bartolomeo, y perdent la vie. Pietro da Rasiglia, le châtelain félon, commet cependant la maladresse de laisser s'échapper le cadet, Corrado III. Ce dernier, qu'une chronique locale désigne quelques années plus tard comme «l'écorcheur de Foligno »" se venge d'une façon terrible. Le donjon de Nocera est assiégé. Pietro se suicide alors que tout son entourage est exécuté. C'est sur cette trame commune que les chroniqueurs brodent leur récit, avec parfois d'importantes divergences.

Pour tenter d'éclairer la mise en scène de la violence et son interprétation dans les textes qui ont pu être rassemblés, deux moments ont été retenus. Il faut tout d'abord chercher les échos de l'épisode, dans le temps et dans l'espace, avant de se concentrer sur deux développements particuliers. Dans ceux-là en effet, la reconstruction de la violence poursuit des objectifs radicalement opposés : pour l'un, la dénonciation de la tyrannie ; pour l'autre, la justification de l'usage de la force et du sang versé à des fins politiques.

Alors même que l'Italie centro-septentrionale est ravagée par les guerres et les pillages, le récit de l'épisode de Nocera se diffuse. Les rumeurs qui l'entourent s'enflent et le déforment. Les régions voisines de l'Ombrie, les Marches et la Toscane, en reçoivent l'écho : des chroniqueurs d'Urbino et de Lucques le relatent. Les développements auxquels le double assassinat des Trinci donne lieu et la répression qui l'a suivi sont bien entendu tout à fait variables. À la cour des Montefeltre, un anonyme note ainsi laconiquement :

\footnotetext{
${ }^{3}$ Les Memoriale degli Unti évoquent « Corrado [...] che allora vi era Signore o per dir melio scorticatore ». La chronique de Pietruccio degli Unti couvrait la période 1424-1440. Le texte original est perdu mais il est partiellement connu grâce à des copies réalisées aux XVII ${ }^{\mathrm{e}}$ et XVIII ${ }^{\mathrm{e}}$ siècles. Voir Michele FAloci Pulignani (éd.), Fragmenta Fulginatis Historiae, dans Rerum Italicarum Scriptores, t. XXVI, Partie II, Bologne, Zanichelli, 1933, p. 29-32. La citation provient du récit de la chute de la seigneurie en 1439, p. 39.
} 
1421 die VIII januarii. Fo la novità in la terra de Nocera contro Nicolò et Bartholomeo de Trinci alora morti etc. et inmediate ne seguì la morte del Castellano de quel loco et de più de cento persone ${ }^{4}$.

Les récits que l'on trouve dans les cités ombriennes sont plus étoffés. Les circonstances font l'objet de longs développements. Les deux auteurs de Gubbio et de Spolète, Ser Guerriero di Ser Silvestro de' Campioni et Parruccio Zampolini, font, comme l'anonyme d'Urbino, œuvre de chroniqueur. Ils se montrent soucieux d'indiquer le degré de véracité qu'ils accordent à leur propre récit et précisent qu'ils relatent les nouvelles qui circulent. Zampolini note ainsi que «l'on dit» (fo dittu) que Nicolò de Ugolino delli Trinci da Foligni a trouvé la mort le deuxième jour de janvier, et précise peu après que «l'on raconte » (fo dictu) que les faits ont eu lieu lors d'une partie de chasse dans les environs de Nocera $^{5}$. Ser Guerriero quant à lui, alors qu'il expose les motifs du geste homicide de Pietro da Rasiglia, écrit : fo dicto l'havea facto perchè uno de quelli signori usciva con la moglie . $^{6}$

Ce sont les représailles conduites par Corrado qui occupent la plus grande part des récits. Les chroniqueurs laissent une large place aux détails sanglants et à la violence de la répression. Parruccio Zampolini raconte ainsi que la famille

\footnotetext{
4 « Le huitième jour de janvier, la nouvelle nous parvint qu'en la terre de Nocera, Nicolò et Bartholomeo Trinci venaient d'être assassinés. Il s'ensuivit sur-le-champ la mort du châtelain du lieu et de plus de cent personnes ». Voir Giovanni ScATENA (éd.), Cronachetta di Urbino, 1404-1444, Urbino, QuattroVenti, 1995, p. 26. La trace de cet événement se trouve dans un court texte, qui couvre six feuillets. Pour comprendre cette mention par l'auteur anonyme, fonctionnaire de la chancellerie selon Giovanni Scatena, il faut esquisser les liens qui unissent les cours de Foligno et d'Urbino. Le grand-oncle de Corrado, Corrado II, qui domine la cité de 1377 à 1386, a épousé Anna da Montefeltre. Lorsqu'il se rend à Rome en 1424 pour le mariage du prince de Salerne, neveu de Martin V, Corrado III est accompagné par le comte de Montefeltro, Guidantonio. Voir sur ce point Maria Virginia PROSPERI VALENTI, «Corrado Trinci, ultimo signore di Foligno », Bollettino della Deputazione di Storia Patria per l'Umbria, LV (1958), p. 105. Le peintre de cour des Montefeltre, Ottaviano Nelli, travaille au même moment à la décoration de la chapelle palatine de Foligno.

${ }^{5}$ Voir Achille SANSI (éd.), «Frammenti degli Annali di Spoleto di Parruccio Zampolini », Documenti storici inediti in sussidio allo studio delle memorie umbre, partie I e II, Foligno, Sgariglia, 1879, p. 159.

6 «On raconte qu'il agit ainsi car l'un des seigneurs couchait avec son épouse ». Voir Giuseppe MAzZATINTI (éd.), «Cronaca di Ser Guerriero di Ser Silvestro de’ Campioni da Gubbio », Archivio Storico per le Marche e per l'Umbria, t. I, 2 (1884), p. 415.
} 
de Pietro a été traquée à travers le territoire de la cité et que, ramenée à Foligno, elle y a été exterminée. Le quartier où ont eu lieu les exécutions baigne alors dans le sang :

Fu dictu che inter maschi, femine vechi et juveni et femine gravide, fuoru più de cinquanta li morti per questa vendetta, sicché alcunu disse che in grande spatiu della dicta contrada de Fuligne dove questo fo fattu non secce potia dare unu passu che non venesse postu el piede nello sangue, cervella, budella, et mani o membra delli dicti morti ${ }^{7}$.

Avec le temps, la nouvelle se répand et se déforme, les rumeurs les plus sordides se propagent : le nombre des victimes est toujours plus élevé, les conditions de leur assassinat toujours plus cruelles. Les chroniqueurs, imperturbablement, enregistrent ce qui se dit. Zampolini note l'amplification et remarque :

De po' cierti dì et misi venivanu da Fuligni de quilli che eranu stati ad sì fatte occisioni per la vendetta delli dicti signiuri, et più numero diciva chi più de reto veniva et alcuno disse che per scripta fatta per alcunu che li morti chi de ferru chi de focu chi de fame et chi appichati eranu più de trecentu et fo dictu che trentasei bestie asinine inseme givanu per Fuligni carche delli morti perché fusseru ben viduti et alcuna femina gravida dello seme de nocenti secce aspettava fine al partu per uccidere el figliolu che faciva, et como era natu lu facivanu morire de fame ${ }^{8}$.

Le succès de l'épisode ne se dément pas, et les faits deviennent matière littéraire. De nouveaux détails sont ajoutés. Dans les Nouvelles qu'il publie sous

\footnotetext{
${ }^{7}$ «On raconta qu'entre les hommes, les femmes, vieilles ou jeunes, et les femmes enceintes, il y eut plus de cinquante personnes qui trouvèrent la mort du fait de cette vengeance, tant et si bien que certains purent dire qu'à travers le vaste espace de la contrade dans laquelle ces événements eurent lieu, il n'était pas possible de faire un pas au sec, sans mettre le pied dans le sang, la cervelle ou les boyaux, sur les mains ou sur les membres de ceux qui avaient été tués. » Voir Achille SANSI (éd.), "Frammenti degli Annali di Spoleto... », art. cit., p. 161-162 8 «Les jours et les mois passant, arrivaient de Foligno de nouveaux témoins des meurtres perpétrés par vengeance par les dits seigneurs, et le nombre des morts croissait au fil des récits. Quelques-uns disaient, comme si cela était un fait avéré, que les victimes, tuées par le fer ou la faim, brûlées ou écorchées, avaient été plus de trois cents. On raconta que trente-six ânes avaient fait le tour de Foligno chargés des cadavres afin que ceux-ci soient vus de tous, et que l'on avait attendu qu'une femme enceinte accouche afin de tuer le fils qu'elle venait de mettre au monde en le laissant mourir de faim. », ibid., p. 162.
} 
forme de recueil à partir de 1545, Matteo Bandello s'inspire librement des chroniqueurs du siècle précédent. Pour les besoins de la narration et pour toucher le public, la violence et la cruauté sont tout entières reportées sur le félon. Ce dernier, après avoir traîtreusement poignardé Niccolò, traîne Bartolomeo, rebaptisé César pour l'occasion, vers le cadavre émasculé de son frère. Il égorge alors le jeune homme, come un agnello, et le sang de la victime

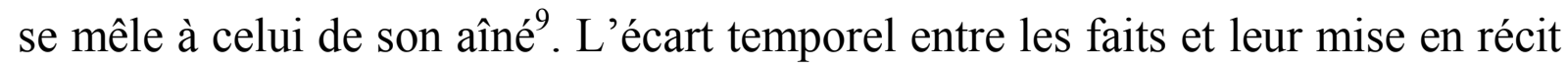
s'est accru et la nature littéraire du texte de Bandello modifie profondément le rôle joué par la violence dans la narration. Mais si les objectifs de l'auteur de nouvelles diffèrent de ceux du chroniqueur, si l'horizon d'attente du lecteur des chroniques $\mathrm{du} \mathrm{Xv}^{\mathrm{e}}$ siècle diverge radicalement de celui de l'amateur de nouvelles du XVI ${ }^{\mathrm{e}}$, la reprise de la trame événementielle montre bien la postérité et la plasticité des faits de $1421^{10}$.

L'amplification et l'exagération dont l'histoire du massacre fait l'objet ne sont pas de simples phénomènes narratifs et ne sauraient être réduites à un supposé attrait pour le morbide. Elles prennent place dans un contexte plus vaste, celui de la réflexion que mène une société sur la légitimité de la violence. Les torts sont en effet partagés. Ser Pietro a fait preuve de traîtrise et de duplicité, Corrado de cruauté. Les familles ennemies sont renvoyées dos à dos par le chroniqueur de Spolète lorsqu'il esquisse les origines familiales du châtelain de Nocera. Zampolini laisse en effet supposer, ce que confirme le Lucquois Giovanni Sercambi, que le père de Pietro exerçait une charge au nom des Trinci et qu'abusant de son autorité, il s'est, avec la complicité de ses maîtres, considérablement enrichi :

\footnotetext{
${ }^{9}$ La nouvelle est indiquée par Arnoldo Picuti, qui a récemment publié dans un petit recueil destiné au grand public trois récits de l'épisode de Nocera : ceux de Giovanni Sercambi, Matteo Bandello et Parruccio Zampolini. Voir Arnoldo Picuti, La strage di Nocera. La vendetta dei Trinci, Foligno, Edicit, 2004, p. 48-63, pour la nouvelle de Bandello.

${ }^{10} \mathrm{Au} \mathrm{XX}{ }^{\mathrm{e}}$ siècle, ceux-ci constituent encore l'intrigue d'une pièce de théâtre écrite par Ferdinando Merli, I Trinci. Tragedia storica in tre atti, Foligno, Torquato Sbrozzi \& figlio, 1926. Un grand merci à madame Fabrizia Paoletti de la Biblioteca comunale di Foligno, qui a eu la gentillesse de me transmettre les références précises de cet ouvrage.
} 
[ser Pasquale] venne tanto in gratìa delli Signiuri per suoe executioni martorij, asasinamenti, homicidia et altru malfare per piacere delli Signiuri, che fece richeza $[\ldots]^{11}$.

Sa fortune était telle, poursuit le chroniqueur, qu'elle a fini par susciter la jalousie du seigneur de Foligno, qui a banni Pasquale pour s'emparer de ses biens. À l'offense faite au père vient s'ajouter le déshonneur causé par l'adultère. Comme Guerriero de' Campioni, Parruccio Zampolini relate la liaison cachée de Niccolò avec la femme de Pietro. Pour ce dernier, la vengeance est dès lors dictée par la défense de l'honneur. La longue mise en contexte du meurtre initial, si elle ne permet pas de l'excuser, vient cependant expliquer le complot. Celui-là est commis per consigliu del diabolu dès lors qu'il vise à éliminer non seulement le seigneur adultère mais aussi son frère, et conduit à emprisonner injustement un de leurs parents témoin du crime, Berardo da Varano. Corrado, par l'ampleur et la nature de la répression qu'il conduit, sort lui aussi du cadre légitime de la violence. La reprise par la rumeur du thème du meurtre de la femme enceinte témoigne du caractère inacceptable de la violence déployée à Nocera et à Foligno. Celle-là n'est plus licite car elle est sauvage et débridée. Ce que les récits des contemporains mobilisent ainsi, c'est l'image même de l'innocence qu'est l'enfant nouveau-né et ce contre quoi aucune violence, pas même celle, légale, du bourreau, ne saurait être exercée, la femme enceinte ${ }^{12}$. L'intégration et l'amplification de ces deux motifs dans la mémoire des faits viennent estampiller la vengeance de Corrado comme bestiale et, partant, illégitime.

Pour les Trinci comme pour la famille de Pietro, en effet, le recours à une violence, strictement codifiée et mise en œuvre collectivement, s'imposait. Ce

11 « [ser Pasquale] parvint tant et si bien à entrer dans les bonnes grâces des Trinci grâce aux exécutions sommaires, assassinats, homicides et autres méfaits qu'il commit pour plaire à ces seigneurs, qu'il accumula une grande richesse [...]. », dans Achille SANSI (éd.), "Frammenti degli Annali di Spoleto... ", art. cit., p. 160.

${ }^{12}$ Voir Claude GaUVARD, Violence et ordre public au Moyen Âge, Paris, Picard, 2005, p. 15. 
n'est qu'à cette condition que l'honneur bafoué du lignage pouvait être réparé. Les récits provenant de Lucques ou Spolète convergent et mettent en scène des réactions identiques dans les deux parentèles: Pietro agit avec l'aide de son frère Nanni et de son neveu ; Corrado se venge grâce à l'appui de son puissant beau-frère, le condottiere Braccio da Montone. C'est le groupe tout entier qui doit réagir pour affirmer sa cohésion et sa puissance. La violence est constitutive de l'ordre social. La noblesse se définit par le partage d'un ensemble de codes et d'un mode de vie, au sein duquel la violence occupe une place centrale. La revendication de l'appartenance à l'élite passe dès lors par l'adoption de comportements violents qui confirment tout autant l'intégration d'un individu à un groupe donné que la place de ce dernier au sommet de la hiérarchie de la société. Garant de la renommée et du prestige personnel, la violence est pour l'individu la condition indispensable de l'existence sociale ${ }^{13}$. La noblesse entend par ailleurs affirmer son existence même par le droit qu'elle revendique à utiliser la violence ${ }^{14}$. À Nocera cependant, loin de resserrer le tissu social, la violence l'a menacé. Elle est alors discréditée et nommée « cruauté ». Ser Guerriero de' Campioni commente les exécutions ordonnées par Corrado à travers tout le territoire en disant qu'elles ont eu lieu avec grandissima cordelta ${ }^{15}$. La dénonciation de la violence n'a pas seulement une portée individuelle ou sociale, elle a des résonances politiques.

La violence est en effet située au cœur des mécanismes du pouvoir. Elle entre en jeu dans la contestation de ce dernier comme dans sa conservation. Le terme même de « violence » n'apparaît pas dans les textes rassemblés autour de

${ }^{13}$ Claude GAUVARD, Violence et ordre public..., op. cit., p. 282.

${ }^{14}$ À la fin du XIII ${ }^{\mathrm{e}}$ siècle, la législation communale s'efforce d'encadrer la violence des magnats et de la restreindre à la seule forme admise de tous, celle de la vendetta dans laquelle la défense de l'honneur du lignage est mise en jeu. Voir Jean-Claude MAIRE ViguEUR, Cavaliers et citoyens. Guerre, conflits et société dans l'Italie communale, XII ${ }^{\mathrm{e}}-\mathrm{XIII}{ }^{\mathrm{e}}$ siècles, Paris, Éditions de l'École des Hautes Études en Sciences Sociales, 2003, p. 328-330.

15 « une très grande cruauté ». Giuseppe MAZZATINTI (éd.), «Cronaca di Ser Guerriero... », art. cit., p. 415. 
l'épisode de 1421. Plus généralement, il est presque absent des chroniques médiévales occidentales ${ }^{16}$. Lorsque la notion est manifeste, c'est, comme dans le cas de la chronique eugubienne, sous la forme de la «cruauté ». Ce dernier mot ne reçoit pas d'acception morale ou affective. Il appartient avant tout au lexique du politique, car la cruauté « qualifie l'action tyrannique $»^{17}$.

Les enjeux de cette dénomination apparaissent clairement dans un cinquième texte rédigé autour de 1458 à Pérouse, La geste de Braccio Fortebracci $^{18}$. Son auteur, Giovanni Antonio Campano, occupe la chaire de rhétorique de la ville. Protégé des seigneurs du lieu, les Baglioni, il est chargé d'écrire la vie du condottiere qui a placé la cité pérugine au cœur d'un puissant mais éphémère État territorial ${ }^{19}$. Campano consacre un long développement à l'épisode de Nocera. Cela peut sembler paradoxal puisque le héros du récit, Braccio, n'y apparaît que comme l'instrument de la vengeance de Corrado et se trouve cantonné au rôle de personnage secondaire. L'auteur désigne systématiquement les Trinci comme des tyrans et ne manque pas de rappeler l'adultère de Niccolò. Dès lors, les événements de 1421 jouent un double rôle dans l'économie du récit de la vie du grand condottiere. Par contraste, l'épisode permet de désigner comme légitime la domination territoriale de celui qui est fait prince de Capoue par Jeanne II d'Anjou en 1423. Ce sens est confirmé chez Campano quand Pietro da Rasiglia essaye de donner une justification politique à son acte. Alors qu'il entrevoit les conséquences auxquelles le double meurtre qu'il vient de commettre va l'exposer, il tente de rassembler la population de Nocera derrière lui et de la soulever, ad libertatem recuperandam, contre

\footnotetext{
16 Joseph MorSel, "Violence », dans Claude GAUVARD, Alain DE LIBERA, Michel ZINK

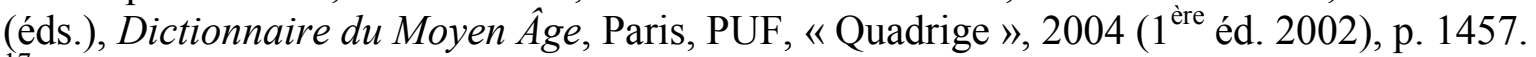

${ }_{17}^{17}$ Claude GAUVARD, Violence et ordre public..., op. cit., p. 12.

${ }^{18}$ Giovanni Antonio CAMPANO, Braccii Perusini Vita et Gesta. Ab anno MCCCLXVIII usque ad MCCCCXXIV, R. VALENTINI (éd.), Rerum Italicarum Scriptores, t. XIX, parte IV, Bologne, Nicola Zanichelli, 1929.

${ }^{19}$ Frank Rutger HausmanN, «Campano, Giovanni Antonio », dans A. GHISALBERTI (éd.), Dizionario Biografico degli Italiani, Rome, Istituto della Enciclopedia Italiana, XVII, 1974, p. 424.
} 
l'oppression des $\operatorname{Trinci}^{20}$. Bien que l'auteur n'adopte pas l'interprétation par laquelle le félon essaye de légitimer ses agissements, il rappelle en la mentionnant que la légitimité de la seigneurie des Trinci n'est pas incontestable. D'autre part, et contrairement au tyran qui le paye de sa vie, Braccio est décrit comme un homme de parole. La duplicité et l'adultère de Niccolò Trinci s'opposent au comportement de Braccio qui respecte ses obligations et honore ses alliances. Chez Campano, l'épisode de Nocera est immédiatement précédé de celui du mariage du condottiere avec Nicolina da Varano, la sœur du Berardo que Pietro retient prisonnier ${ }^{21}$. Le héros n'hésite pas à s'exposer au danger en volant immédiatement au secours de son parent dès qu'il a vent de sa mésaventure $^{22}$. Par contraste avec Braccio, les Trinci apparaissent, politiquement et moralement, comme des seigneurs illégitimes ${ }^{23}$. Ils sont désignés ainsi par leur propre violence. Dans le récit, la cruauté de la répression rend manifeste la tyrannie. L'auteur associe explicitement les deux notions lorsqu'il décrit les cadavres des victimes, démembrés et exposés à travers la ville, témoins de la « cruauté perfide et tyrannique » de Corrado $^{24}$.

\footnotetext{
${ }^{20}$ Giovanni Antonio CAMPANO, Braccii Perusini Vita et Gesta, op. cit., p. 154. La mauvaise foi de Pietro est dénoncée par le narrateur comme par les habitants de Nocera, qui refusent de suivre le châtelain. Cette interprétation en termes de tyrannicide, par les acteurs ou par les auteurs, n'apparaît, au $\mathrm{XV}^{\mathrm{e}}$ siècle, que chez Campano. Elle est ensuite reprise par Matteo Bandello.

${ }^{21}$ Cf. supra, p. 68.

${ }^{22}$ Les liens qui unissent les trois familles sont bien plus étroits que ce Campano en veut bien dire. Ce dernier ne mentionne que les parentés qui servent à l'élaboration d'une image héroïque de Braccio. Or, le condottiere a marié son fils naturel, Oddo, à Elisabetta, l'une des filles de Niccolò Trinci. Niccolò a lui épousé Tora da Varano, sœur de Berardo, alors que ce dernier a pris pour femme la propre sœur du seigneur de Foligno, Viviana Trinci. La femme de Braccio, Nicolina da Varano, est donc la belle-sœur de Niccolò Trinci, lui-même beau-père du fils du seigneur de Pérouse. C'est d'ailleurs à la demande de Corrado Trinci, et non pour délivrer Berardo da Varano, que Braccio assiège le château de Nocera selon les chroniqueurs de Lucques et de Spolète, Giovanni Sercambi et Parruccio Zampolini.

${ }^{23}$ La duplicité de Niccolò Trinci est une tare morale. Un tel défaut a une résonance politique dès lors qu'il rend indigne celui à qui il est imputé de l'exercice de charges publiques ou de responsabilités au sein de la cité.

24 «suae perfidiae et tyrannicae crudelitatis ». Voir Giovanni Antonio CAMPANO, Braccii Perusini Vita et Gesta, op. cit., p. 155.
} 
Si la violence excessive dénonce le tyran, elle n'en conserve pas moins un statut ambigu. Dans toute son horreur en effet, la répression des Trinci peut apparaitre comme un acte politique nécessaire. C'est du moins le sens véhiculé par la dernière chronique qu'il convient d'étudier, celle de Giovanni Sercambi ${ }^{25}$. Dans ce récit, la violence et la cruauté sont mises en scène de façon à acquérir une portée exemplaire. À la différence des textes de Gubbio, de Spolète ou de Pérouse, la chronique lucquoise ne mentionne pas l'adultère. La félonie de Pietro s'en trouve aggravée : le double assassinat n'est plus la réparation d'un affront personnel. Sercambi ne mentionne que les différends qui ont opposé les da Rasiglia aux Trinci $^{26}$ : le père de Pietro et toute sa famille ont été bannis par Ugolino, le père de Niccolò. Lorsqu'il est parvenu au pouvoir, Niccolò a gracié les condamnés et les a réintégrés dans son entourage. Giovanni Sercambi a exercé les plus hautes charges au sein de la commune de Lucques avant de se rallier à la puissante famille des Guinigi et de favoriser l'instauration de leur seigneurie $^{27}$. Durant la première moitié des années 1390, il a rédigé à l'intention de ses protecteurs une Note, véritable programme de gouvernement seigneurial $^{28}$. Celle-ci doit permettre aux nouveaux maîtres de la ville de maintenir leur domination et de préserver l'indépendance d'une cité qui ne s'est arrachée à la tutelle pisane que trente ans auparavant et qui reste menacée par les ambitions florentines et milanaises. La Note aux Guinigi met l'accent sur la nécessité de s'assurer que les plus hautes charges publiques soient attribuées à des hommes personnellement liés au pouvoir en place. L'amitié et la fidélité d'homme à homme sont définies comme les fondements du pouvoir seigneurial. Au fil de la deuxième partie de ses Chroniques, dans laquelle le récit de Nocera

\footnotetext{
${ }^{25}$ Le Croniche di Giovanni Sercambi lucchese, S. Bongi (éd.), 3 volumes, Rome, Istituto Storico italiano (Fonti per la storia d'Italia), 1892. Pour l'épisode de Nocera, t. III, p. 266-273. ${ }^{26}$ Cf. supra, p. 67.

${ }^{27}$ Voir Giovanni SercAmBI, Novelle, G. Sinicropi (éd.), vol. II, Bari, Laterza, 1972, p. $762-$ 768.

${ }^{28}$ Ibid., p. 769 ; voir aussi : « Nota a voi Guinigi nobilibus et potentibus viris Dino, Michaele, Lazzarino et Lazario de Guinigiis », dans Claudio VARESE (éd.), Prosatori volgari del Quattrocento, Milan-Naples, Ricciardi, 1955, p. 125-133.
} 
est intégré, Sercambi multiplie les chapitres intitulés « Nota ». Ce sont autant de conseils de gouvernement nommément adressés aux seigneurs et aux princes du temps, de Gabrielle Maria Visconti, seigneur de Pise, à la reine de Naples. Le passage narratif consacré aux événements de 1421 remplit une fonction identique et fonctionne comme un avertissement aux Guinigi ${ }^{29}$. L'exorde est explicite :

Ora al presente si conterà quanto ogni signoria, comune, stato, persone $\mathrm{e}$ ciascuno si de'guardare e avere avertenzia di non fidarsi di coloro che sono stati contrarii di tal signoria, comune, stato e persone. E perché se n'abbia alcuna experiensia si racorda quello ch'è scripto, dove in questa parte raconta, dicendo : in nemico reconciliato ne confidas in eternum ${ }^{30}$.

L'absence de référence à l'adultère et la dimension exemplaire que doit prendre le récit renversent la signification de la répression. La violence devient nécessaire. Elle est utilisée comme un instrument de gouvernement servant à compenser le manque de prudence dont Niccolò a fait preuve. Dans un mouvement de balancier, la violence vient rééquilibrer une situation politique compromise par un défaut de prévoyance ${ }^{31}$. La mise à mort violente et spectaculaire de ses ennemis contribue à affirmer et à renforcer la seigneurie ${ }^{32}$. Le sort réservé aux parents est évoqué par Sercambi dans un paragraphe à part

\footnotetext{
${ }^{29}$ Cette mise en garde semble d'autant plus susceptible d'atteindre son but que la sœur de Niccolò Trinci, Iacoppa, a épousé un an avant le massacre Paolo Guinigi, seigneur de Lucques.

30 «On racontera ici pourquoi toute seigneurie, toute commune, tout État ou toute personne doit être sur ses gardes et avoir la prudence de ne pas se fier à ceux qui se sont montrés contraires à la seigneurie, à la commune, à l'État, ou à la personne elle-même. Et afin d'en être averti, il faut se rappeler le précepte, illustré par ce récit, qui dit : in nemico reconciliato ne confidas in eternum [Ne te fie jamais à un ennemi repenti]. "Voir Le croniche di Giovanni Sercambi lucchese, op. cit., p. 266.

${ }^{31}$ Le croniche di Giovanni Sercambi lucchese, op. cit., p. 267.

32 Voir Andrea ZORZI, «Rituali di violenza, cerimoniali penali, rappresentazioni della giustizia nelle città italiane centro-settentrionali (secoli XIII-XV) », dans P. CAMMAROSANO (dir.), Le forme della propaganda politica nel Due e nel Trecento, Collection de l'École française de Rome 201, Rome, École française de Rome, 1994, p. 413.
} 
intitulé Nota delle iustitie che si faranno de mali fattori ${ }^{33}$. Les victimes, dont le nombre a été réduit à quatre-vingts, sont des donne, fanciulle et fanciulli, homini et donne gravide ${ }^{34}$, et, l'auteur ajoute immédiatement, così quelli con colpa come quelli senza colpa ${ }^{35}$. Mais comment peut-il se trouver des mali fattori senza colpa? Le chroniqueur semble faire sien le principe de qualification juridique des faits : la mise à mort établit, a posteriori, la culpabilité de la victime. Celle-ci est coupable parce qu'elle a été reconnue comme telle et condamnée. L'exécution désigne celui qui a commis la faute, soit par la part active qu'il a prise au double assassinat, soit par la vengeance qu'il risque de vouloir exercer ou faire exercer à l'encontre des Trinci en tant que membre du lignage de Pietro. Dans un cas comme dans l'autre, c'est un malfaiteur car il constitue une menace contre la stabilité du régime ${ }^{36}$.

La violence n'est pas seulement nécessaire au maintien de la famille dominante et à sa propre définition comme groupe noble. Elle est indispensable à la communauté politique tout entière, car le félon a commis une double faute en rompant le lien personnel de fidélité qui l'unissait à son protecteur. Il a d'abord menacé les conditions mêmes de l'union et de la concorde des citoyens en faisant pénétrer la défiance au cœur de la cité. Il s'est d'autre part attaqué au seigneur détenteur de l'autorité publique, au représentant du corps civique. Dans la visée politique qui est la sienne, Sercambi est le seul à consacrer un développement au sort fait aux cadavres des victimes. La violence imposée aux

33 «Note sur les châtiments infligés aux malfaiteurs », Le croniche di Giovanni Sercambi lucchese, op. cit., p. 273.

34 « des femmes, des jeunes filles et de petits garçons, des hommes et des femmes enceintes », ibid.

35 « ceux qui avaient commis une faute comme ceux qui n'en avaient pas commise », ibid.

${ }^{36}$ La faible étendue du lignage de Pietro et son enracinement hors les murs de Foligno rendent possible la tentative d'extermination de tous les membres de la famille da Rasiglia. Corrado Trinci dispose des moyens nécessaires à l'élimination de quelques dizaines de personnes, sans pour autant risquer de raviver une lutte de factions à l'intérieur même de la cité. La mise à mort de l'ensemble de la parentèle prévient tout risque de résurgence d'un conflit vieux, selon les chroniqueurs cités, de deux générations. 
morts vient redoubler celle des exécutions ${ }^{37}$. Les corps ne sont plus seulement, comme chez Parruccio Zampolini, laissés un temps dans la rue sans sépulture, en signe d'infamie. Sercambi évoque la violence rituelle qui leur est imposée : ils sont réduits à l'état de carcasses de boucherie, démembrés, et offerts aux chiens qui se repaissent des restes. À Bassano, en 1373, les cadavres de quatre traîtres subissent un sort comparable : découpés en morceaux par des bouchers, ils sont finalement offerts aux loups. Le corps du tyrannicide de Galeazzo Maria Sforza, en 1476, est, quant à lui, laissé aux cochons. Rabaissé à l'état animal, le corps du traître peut ainsi être, par ce rituel réparateur, extirpé du corps social avant de disparaître sans laisser de trace ${ }^{38}$. Le chroniqueur lucquois mentionne un deuxième traitement. Les corps mutilés sont exposés et les membres arrachés placés à travers la ville, sur les places, les portes, les murailles. À travers tout l'espace civique, le pouvoir affiche le sort réservé à ceux qui ont menacé l'unité de la communauté placée sous la tutelle seigneuriale.

Les regards croisés des chroniqueurs sur l'épisode de Nocera ont permis de considérer la violence comme un élément structurant de la société italienne de la fin du Moyen Âge. Elle délimite un groupe social, constitue le moyen de maintenir l'ordre politique, ou, lorsqu'elle est présentée comme excessive, vient légitimer la contestation de ce dernier. Mais ce qui s'est fait jour au fil de cette étude, est avant tout l'enjeu de la dénomination et de la qualification des faits rapportés. Nommer, c'est mettre en forme et articuler la diversité du réel. Les chroniqueurs du massacre de 1421 sont bien conscients du pouvoir des mots et de leur capacité à fixer la mémoire, immédiate ou à venir. Ils entreprennent selon les buts qu'ils recherchent, de définir la tonalité de l'événement pour les temps futurs.

\footnotetext{
37 Campano mentionne lui en quelques mots les cadavres démembrés, mais c'est pour interpréter immédiatement la vengeance comme un nouveau signe de cruauté et de tyrannie.

38 Voir Andrea ZORZI, «Rituali di violenza, cerimoniali penali...», art. cit., p. 401. Les exemples de Bassano et de Milan sont empruntés à cet auteur.
} 